\title{
ANÁLISE DO MÉTODO DO PERMEÂMETRO GUELPH NA DETERMINAÇÃO DA CONDUTIVIDADE HIDRÁULICA SATURADA
}

\author{
An Analysis of Method of Guelph Permeameter in Estimating Saturated Hydraulic Conductivity
}

Miguel Alfaro Soto ${ }^{1}$, Kiang Hung Chang ${ }^{1}$ e Orencio Monje Vilar ${ }^{2}$

\begin{abstract}
RESUMO: $\quad$ O permeâmetro Guelph tem sido empregado amplamente na determinação da condutividade hidráulica medida in situ $\left(\mathrm{K}_{\mathrm{f}}\right)$, apresentando comumente resultados negativos da condutividade. Embora estes resultados sejam atribuídos geralmente à heterogeneidade do solo, outros fatores inerentes a este método podem causar estes valores. O principal objetivo deste trabalho é analisar o método do permeâmetro Guelph na determinação da condutividade hidráulica saturada em um solo arenoso e outro argiloso, empregando os métodos de um e dois estágios. As análises evidenciaram as dificuldades de cumprir a condição de isotropia de $\mathrm{K}_{\mathrm{fs}}$ no método de dois estágios, porém, o uso desta técnica é possível com a minimização de erros operacionais. Em contrapartida, o método de um estágio poderá ser aplicado visando a determinação aproximada de $\mathrm{K}_{\mathrm{fs}} \mathrm{e}$ até com um número reduzido de ensaios. No entanto, a escolha adequada do parâmetro $\alpha$, assim como da altura $(\mathrm{H})$, pode minimizar erros indesejáveis na sua determinação.
\end{abstract}

Palavras-chave: Permeâmetro Guelph, Condutividade Hidráulica Saturada, Métodos de um e dois estágios

\begin{abstract}
The use of the Guelph permeameter method has, in many situations, shown to provide unrealistic results such as negative values of the hydraulic conductivity $\left(\mathrm{K}_{\mathrm{fs}}\right)$. Although this is attributed essentially to soil heterogeneity, other factors inherent to the method itself can also interfere with the results. The primary objective of the present work is to analyze the performance and the variability of the Guelph permeameter in determining the hydraulic conductivity of sandy soils and other loamy soils when the one-stage or two-stage load method is employed. Inherent difficulties in meeting with the $\mathrm{K}_{\mathrm{fs}}$ condition of isotropy in the two-stage load method are evidenced. Notwithstanding, the use of the two-stage load method is still possible when operational errors are minimized. In compensation, the one-stage method is employed in determining only the saturated hydraulic conductivity and only a reduced number of tests is needed. However, the appropriate choice of the parameter $\alpha$ and the height $(\mathrm{H})$ can minimize the errors associated to estimating $\mathrm{K}_{\mathrm{fs}}$.
\end{abstract}

Key words: Guelph Permeameter, saturated hydraulic Conductivity, single and two constant head well permeameter method.

\section{INTRODUÇÃO}

A medição da condutividade hidráulica in situ na zona não saturada ou vadosa é empregada em investigações de diversas áreas do conhecimento. $\mathrm{Na}$ hidrogeologia e geotecnia, pode ser utilizada em estudos de avanços de frente de infiltração em taludes, projetos de canais e reservatórios, modelagem de balanços hidrológicos e de transporte de solutos, entre outros.

Vários são os métodos disponíveis na literatura especializada destinados à quantificação da condutividade hidráulica in situ, e a escolha adequada de um determinado método dependerá das características do solo e das condições do local a ser estudado (BANTON, 1993). O método do permeâmetro Guelph talvez seja um dos de maior aceitação e pode ser classificado, entre outros métodos, como o mais rápido no procedimento de ensaio, fácil emprego e cálculo da condutividade hidráulica, de maior exatidão e de menor perturbação no solo (RAGAB e COOPER, 1990).

Consiste em um permeâmetro de carga constante que trabalha sob o princípio do tubo de Mariotte, e permite determinar entre outras propriedades (potencial matricial de fluxo e sorção) a condutividade hidráulica saturada, levando em consideração o efeito do fluxo não saturado.

A determinação da condutividade hidráulica comumente é realizada segundo procedimentos de ensaio e cálculos denominados de um estágio(ELRICK et al., 1989), mantendo uma altura constante de água no furo de sondagem, e dois estágios (REYNOLDS e 
ELRICK, 1985), onde uma segunda e maior altura de água é aplicada após a conclusão do primeiro estágio.

Diversas pesquisas têm mostrado que o método de dois estágios pode fornecer resultados negativos de condutividade hidráulica, ou ainda, do potencial matricial de fluxo. Reynolds e Elrick (1986), Philip (1985), Amoozegar (1989), entre outros, sugerem que esses resultados são causados pela heterogeneidade ou macroporosidade no meio poroso, resultando em características de fluxo diferentes, entre um e outro estágio de medição. Entende-se por heterogeneidade, ou macroporosidade do solo, as camadas com características diferenciadas quanto à compactação, textura, umidade, presença de furos no solo produzidos por raízes, formigueiros, entre outros. A esse respeito, Elrick et al. (1989) sugeriu, para obtenção da condutividade hidráulica saturada e do potencial matricial de fluxo, o emprego do método de um estágio a partir da estimativa inicial da sorção que podem ser encontrada em tabelas na literatura especializada para a maioria dos solos. A justificativa é baseada em resultados de White e Sully (1987) pelo fato de ambos os parâmetros não serem significativamente influenciados pela sorção.

Adicionalmente, o método do permeâmetro Guelph tem sido comparado com outros baseados em técnicas de campo e de laboratório. Entre eles, Lee et al. (1985) mostraram que o permeâmetro Guelph reproduz valores estatisticamente iguais ao permeâmetro de entrada de ar (in situ) e de parede rígida (laboratório), principalmente em solos de textura fina. Bagarello e Provenzano (1996) mostraram resultados estatisticamente iguais (ensaiados em areia siltosa indeformada) com o permeâmetro de parede rígida, em amostras com dimensões significativas $(8,5 \mathrm{~cm}$ de diâmetro e $11 \mathrm{~cm}$ de altura) e em solos relativamente secos (umidade menor que 12\%). Soto (1999) mostrou resultados similares a Bagarello e Provenzano (1996), porém, em solos arenosos e argilosos indeformados típicos do estado de São Paulo e relativamente úmidos (umidade menor que $12 \%$ ). Resultados estatisticamente diferentes foram também reportados; no entanto, as hipóteses para esse comportamento sugerem medição de diferentes sentidos de fluxo (no permeâmetro
Guelph, a condutividade é resultante do fluxo horizontal e vertical e, em outros, apenas vertical), dimensões de solo testado, entre outros.

Este trabalho tem como objetivo analisar o uso do método do permeâmetro Guelph na determinação da condutividade hidráulica saturada de campo e estabelecer critérios para aperfeiçoar o emprego do mesmo devido a algumas limitações reportadas.

\section{MODELOS DE FLUXO PARA ENSAIOS EM FUROS DE SONDAGEM}

O método do permeâmetro Guelph foi desenvolvido por Reynolds et al. (1983) para determinar in situ a condutividade hidráulica saturada de campo $\left(\mathrm{K}_{\mathrm{fs}}\right)$ e, posteriormente estendida, por Reynolds e Elrick (1985), levando em conta os efeitos do fluxo não saturado. Como consequência do novo modelo teórico, foram também obtidos outros parâmetros adicionais, tais como, o potencial matricial de fluxo e o parâmetro $\alpha$ que serão analisados posteriormente.

O modelo é baseado na equação de Richards (1931), para fluxo permanente em furo cilíndrico, dada pela equação (8):

$$
\mathrm{Q}=\left[\frac{2 \pi \mathrm{H}^{2}}{\mathrm{C}}+\pi \mathrm{a}^{2}\right] \mathrm{K}_{\mathrm{fs}}+\left[\frac{2 \pi \mathrm{H}}{\mathrm{C}}\right] \phi_{\mathrm{Gm}}
$$

Em que $\mathrm{Q}\left[\mathrm{L}^{3} \mathrm{~T}^{-1}\right]$ é a vazão a carga constante, $\mathrm{H}$ [L] é a altura de água constante no furo de sondagem; $\mathrm{K}_{\mathrm{fs}}\left[\mathrm{LT}^{-1}\right]$ é a condutividade hidráulica saturada medida in situ, a [L] é o raio do furo de sondagem, $\phi_{\mathrm{Gm}}\left[\mathrm{L}^{2} \mathrm{~T}^{-1}\right]$ é o potencial matricial de fluxo e C [-] um parâmetro denominado "fator de forma", que depende da relação $\mathrm{H} / \mathrm{a}$ e do tipo de solo. Graficamente, a Soilmoisture Equipment Corp. (1986) fornece graficamente o fator $C$ para três classes de solos (textura e macroporosidade).

Esses parâmetros foram deduzidos a partir das superfícies de dois bulbos que se formam no solo ao redor do furo de sondagem, durante a execução de um ensaio (ELRICK et al., 1989) e que está esquematizado na Figura 1. 


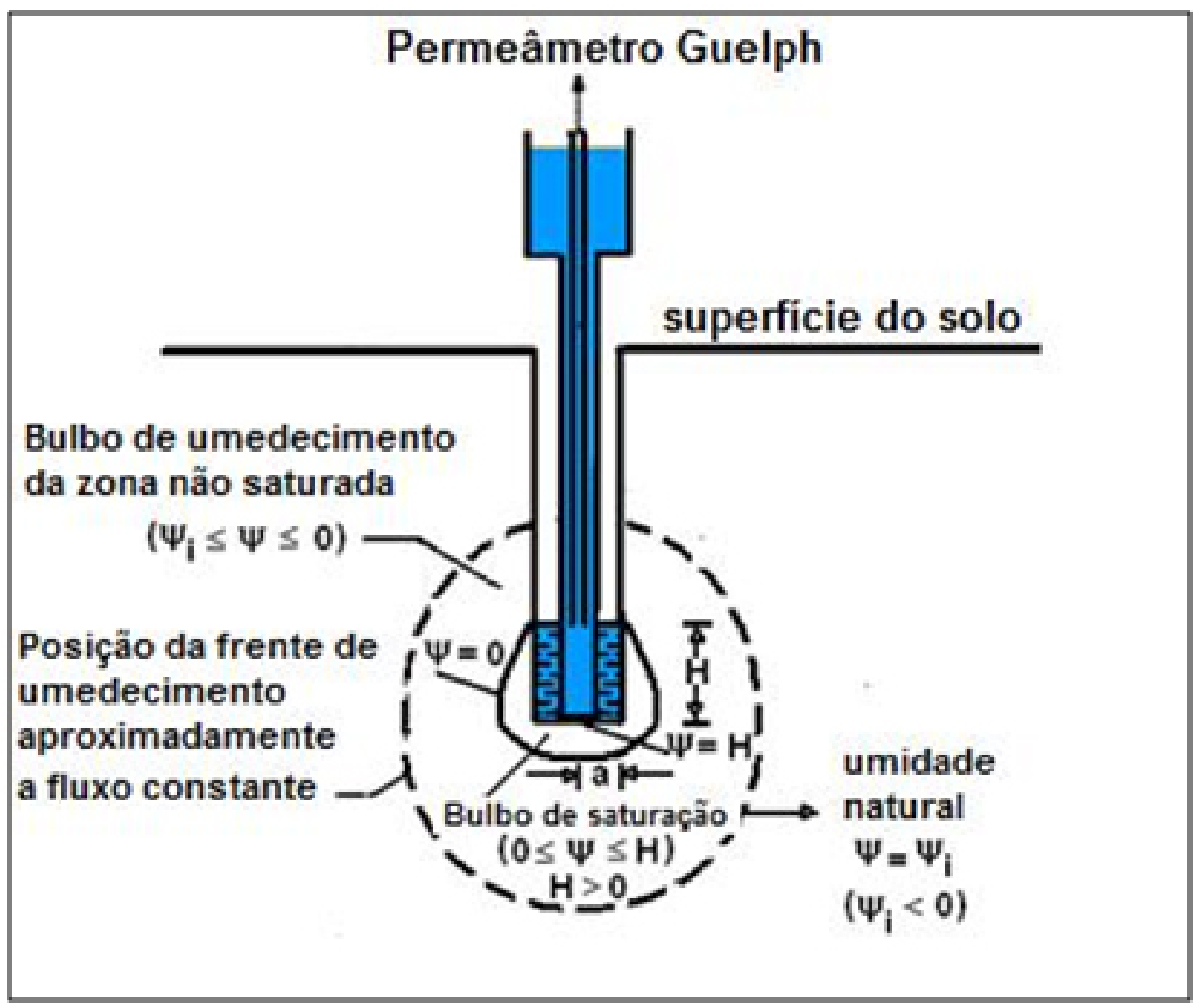

Figura 1: Bulbo saturado e não saturado ao redor do furo de sondagem durante o ensaio a carga constante. Figure 1: Saturated and unsaturated bulb around the base of a test hole during a constant head test.

Segundo Elrick et al. (1989), durante o ensaio, o bulbo úmido, porém não saturado (bulbo maior), migra e expande a partir do bulbo saturado (bulbo menor) onde a vazão Q é representada pelas duas parcelas contidas na equação (1), sendo que a primeira corresponde ao fluxo saturado e a segunda ao fluxo não saturado (REYNOLDS e ELRICK, 1985).

O fluxo não saturado é influenciado principalmente pelo potencial matricial de fluxo e que foi definida a partir de Gardner (1958):

$$
\phi_{\mathrm{Gm}}=\int_{\psi_{\mathrm{i}}}^{0} \mathrm{~K}(\psi) \mathrm{d} \psi \quad \Psi_{\mathrm{i}} \leq \Psi \leq 0
$$

Em que $\Psi$ [L] é a carga de pressão (neste caso a sucção) de água no solo e $\mathrm{K}(\Psi)\left[\mathrm{LT}^{-1}\right]$ é a função da condutividade hidráulica. Nesta equação, o limite superior da integração $(\Psi=0)$ e o inferior $\left(\Psi=\Psi_{i}\right)$ referem-se aos limites do bulbo saturado e não saturado, respectivamente, como é mostrado na Figura 1. Dentro do bulbo saturado, a pressão de água no solo é $\Psi>0$ e dentro do bulbo não saturado, $\Psi<0$, que corresponde à sucção do solo.

Inicialmente, a equação (2) foi utilizada por Gardner(1958) como um artifício de transformação para linearizar a equação diferencial corresponde à equação de Richards (1931) obtendo uma equação empírica exponencial, utilizada por Reynolds e Elrick (1985) para determinar a função condutividade hidráulica não saturada, cuja solução para as condições de contorno ilustradas na Figura 1 pode ser expressa como:

$$
\mathrm{K}(\psi)=\mathrm{K}_{\mathrm{fs}} \mathrm{e}^{\alpha \psi} \quad \alpha>0 ; \psi \leq 0
$$

Onde: $\mathrm{K}_{\mathrm{fs}}$ condutividade hidráulica saturada medida in situ $\left[\mathrm{LT}^{-1}\right]$; e: 2,71828 ; $\psi$ :corresponde à sucção do solo; $\alpha$ : constante $\left[\mathrm{L}^{-1}\right]$ que 
depende das propriedades do solo, principalmente da macroporosidade do solo e, secundariamente, da textura (BRESLER, 1978; REYNOLDS e ELRICK, 1985; ELRICK et al., 1989). Este parâmetro pode ser obtido através do ensaio ou estimado in situ.

A solução analítica da equação (3) descreve a variação da condutividade hidráulica não saturada em função da sucção do solo. Segundo essa função, quando a sucção do solo aumenta, a condutividade hidráulica diminui exponencialmente, a partir do valor máximo $\mathrm{K}_{\mathrm{fs}}(\operatorname{com} \psi \geq 0)$.

$\mathrm{O}$ parâmetro $\alpha$ no método do permeâmetro Guelph pode ser obtido mediante a lei de fluxo proposta por Reynolds e Elrick (1985).

Para isso, a equação (3) pode ser substituída na equação (2), o que resulta:

$$
\phi_{\mathrm{Gm}}=\frac{\mathrm{K}_{\mathrm{fs}}}{\alpha}\left[1-\mathrm{e}^{\alpha \psi_{\mathrm{i}}}\right]
$$

Segundo Scotter et al. (1982), considerando que $\exp \left(\alpha \psi_{\mathrm{i}}\right)<<1$ para solos relativamente secos $\left(\psi_{\mathrm{i}} \leq-10 \mathrm{kPa}\right)$ em estado natural, a expressão pode ser ainda reduzida para:

$$
\alpha=\mathrm{K}_{\mathrm{fs}} / \phi_{\mathrm{Gm}}
$$

Assim, o parâmetro $\alpha$ é reduzido a uma relação entre a condutividade hidráulica saturada de campo $\mathrm{K}_{\mathrm{fs}}$ e o potencial matricial de fluxo $\phi_{\mathrm{Gm}}$, ambos obtidos in situ, através do permeâmetro.

\section{Determinação de $\mathbf{K}_{\mathrm{fs}}, \phi_{\mathrm{Gm}}$ e $\alpha$ com o método do Permeâmetro Guelph}

Para determinar os parâmetros $\left(\mathrm{K}_{\mathrm{fs}}, \phi_{\mathrm{Gm}}\right)$ da solução da equação (1), utilizaram-se os métodos (procedimentos de ensaio e cálculo) de um estágio (ELRICK et al., 1989) e dois estágios (REYNOLDS e ELRICK, 1985).

$\mathrm{O}$ método de um estágio consiste em aplicar uma altura de água $\mathrm{H}$ constante no furo de sondagem e, quando o regime permanente é atingido, a vazão $\mathrm{Q}$ e os parâmetros $\mathrm{K}_{\mathrm{fs}}$ e $\phi_{\mathrm{Gm}}$ são determinados por:

$$
\begin{gathered}
\mathrm{K}_{\mathrm{fs}}=\frac{\mathrm{CQ}}{\left(2 \pi \mathrm{H}^{2}+\pi \mathrm{a}^{2} \mathrm{C}+2 \pi \mathrm{H} / \alpha\right)} \\
\phi_{\mathrm{Gm}}=\frac{\mathrm{CQ}}{\left[\left(2 \pi \mathrm{H}^{2}+\pi \mathrm{a}^{2} \mathrm{C}\right) \alpha+2 \pi \mathrm{H}\right]}
\end{gathered}
$$

O parâmetro $\alpha$ é estimado inicialmente a partir da Tabela 1, onde o valor adotado dependerá da macroporosidade (fissuras, formigueiros, furos de raízes, etc.) e textura do solo.

O método de dois estágios consiste em aplicar duas alturas $H_{i}$ de água sucessivas no furo de sondagem. Uma vez determinadas as vazões para o regime permanente $\mathrm{Q}_{i}$, correspondente a cada $\mathrm{H}$ aplicado, os parâmetros $\mathrm{K}_{\mathrm{fs}}$ e $\phi_{\mathrm{Gm}}$ são obtidos a partir de duas equações simultâneas (Equação 8). O parâmetro $\alpha$ neste método é determinado através da equação (5).

$$
\mathrm{Q}_{\mathrm{i}}=\left(\frac{2 \pi \mathrm{H}_{\mathrm{i}}^{2}}{\mathrm{C}_{\mathrm{i}}}+\pi \mathrm{a}^{2}\right) \mathrm{K}_{\mathrm{fs}}+\left(\frac{2 \pi \mathrm{H}_{\mathrm{i}}}{\mathrm{C}_{\mathrm{i}}}\right) \phi_{\mathrm{Gm}}(8)
$$

Onde: $\mathrm{C}_{\mathrm{i}}\left(\mathrm{C}_{2}>\mathrm{C}_{1}\right), \mathrm{H}_{\mathrm{i}}(\mathrm{H} 2>\mathrm{H} 1), \mathrm{Q}_{\mathrm{i}}(\mathrm{Q} 2>\mathrm{Q} 1)$.

\section{MATERIAIS E MÉTODOS}

\section{Descrição dos locais de estudo}

O primeiro, dentre os dois materiais estudados, pertence ao campo experimental da Universidade Federal de Santa Catarina na Cidade de Florianópolis-SC, que se denominou como local A, e corresponde a um sedimento cenozóico, representado por depósito arenoso. $\mathrm{O}$ segundo é oriundo do bairro Jardim Medeiros na cidade de São Carlos-SP, denominado como local B, e corresponde ao material residual derivado dos magmatitos básicos da Formação Serra Geral.

Tabela 1: Valores $\alpha$ sugeridos (ELRICK et al, 1989) em função da macroporosidade e textura do solo

Table 1: $\alpha$ suggested (ELRICK et al, 1989) on the basis of structural and textural considerations

\begin{tabular}{|c|c|}
\hline $\boldsymbol{\alpha}\left(\mathbf{c m}^{-1}\right)$ & TIPO DE SOLO \\
\hline 0,01 & Argilas compactas (aterros, liners, sedimentos lacustres e marinhos) \\
\hline 0,04 & Solos de textura fina, principalmente sem macroporos e fissuras. \\
\hline 0,12 & Argilas até areias finas com alta a moderada quantidade de macroporos e fissuras. \\
\hline 0,36 & Areia grossa inclui solos com macroporos e fissuras. \\
\hline
\end{tabular}

\section{Ensaio de Campo com o Permeâmetro Guelph}

Os ensaios foram realizados em ambos os locais numa profundidade de $0,45 \mathrm{~m}$, distribuídos em áreas de aproximadamente $20 \mathrm{~m} \times 50 \mathrm{~m}$.
No local A, 22 ensaios foram conduzidos utilizando o método de um estágio e com nível de água $\mathrm{H}$ constante igual a $2,5 \mathrm{~cm}$. Durante o ensaio, a variação do nível de água no reservatório $(\Delta \mathrm{L})$ do permeâmetro foi monitorada em intervalos de 30 
segundos $(\Delta t)$ até atingir o regime permanente. $\mathrm{O}$ critério adotado para alcançar esse estado consistiu em parar o ensaio quando se observou a repetição do valor mínimo da razão $\Delta \mathrm{L} / \Delta \mathrm{t}$ em quatro medições consecutivas. A determinação de $\mathrm{K}_{\mathrm{fs}}$ e $\phi_{\mathrm{Gm}}$ foi feita a partir das equações (6) e (7), respectivamente. Inicialmente o parâmetro $\alpha$ foi inferido por avaliação visual (com auxílio da Tabela 1), resultando em $0,16 \mathrm{~cm}^{-1}$.

No local B, foram realizados 20 ensaios utilizando o método dos dois estágios, onde as alturas empregadas $\left(\mathrm{H}_{1}<\mathrm{H}_{2}\right)$ foram de 10 e $12 \mathrm{~cm}$, visto que, estas alturas foram as mais adequadas para a vazão existente. No procedimento, a segunda altura $\mathrm{H}_{2}$ foi aplicada, logo depois de atingido o regime permanente no primeiro estágio. A entrada de ar cria obstáculos (aumento da tortuosidade) que provocam a diminuição da velocidade de infiltração no segundo estágio, o que compromete os resultados de $\mathrm{K}_{\mathrm{fs}}$. Em ambas as alturas, a variação do nível de água no reservatório do permeâmetro foi monitorada em intervalos de um minuto, adotando o critério já descrito anteriormente (quatro leituras consecutivas $\Delta \mathrm{L} / \Delta \mathrm{t}$ iguais) para verificar a finalização de cada estágio. A determinação dos parâmetros $\mathrm{K}_{\mathrm{fs}} \mathrm{e} \phi_{\mathrm{Gm}}$ foi realizada com a solução das duas equações geradas a partir de (8) e, uma vez conhecidos, o parâmetro $\alpha$ foi determinado com auxílio da equação (5).

\section{RESULTADOS E ANÁLISES}

Foram realizados ensaios para caracterização dos materiais provenientes dos locais A e B segundo as recomendações das normas ABNT. A esse respeito, a Tabela 2 apresenta uma síntese dos resultados de ensaios de granulometria, classificação textural, índices físicos, bem como, limites de consistência.

Em cada tipo de material foi avaliada a relação Q/Qo (vazão Q por vazão inicial Qo) em função do tempo, com a finalidade de observar qualitativamente a variabilidade dessa relação com o tempo. As Figuras 2 a 4 mostram os resultados obtidos para o material $\mathrm{A}$ $\left(\mathrm{H}_{1}=2,5 \mathrm{~cm}\right)$ e B para o primeiro estágio $(\mathrm{H}=10 \mathrm{~cm})$ e segundo estágio $\left(\mathrm{H}_{2}=12 \mathrm{~cm}\right)$, respectivamente.

Da Figura 2, observa-se que o material A se caracteriza por uma maior variabilidade de resultados em relação ao material B da Figura 3. A variabilidade total em alguns ensaios geotécnicos, tal como apontado por Rétháti (1988), é composta por uma parcela correspondente ao método de medição e outra correspondente à heterogeneidade inerente do solo. Levando em consideração que, em todos os casos, os ensaios foram executados por um único operador, podemos inferir uma heterogeneidade maior ao material arenoso.

Ainda na Figura 2, observa-se um menor tempo (em torno de 3 a 4 minutos) para atingir o regime permanente, por não possuir uma acentuada diminuição de $\mathrm{Q} / \mathrm{Q}$ o com o tempo. Isto é evidente, pois o material arenoso permite uma maior mobilidade da água sendo mais permeável e com menor capacidade de retenção de água nos interstícios e porque, adicionalmente, encontravase próximo à saturação $(\mathrm{Sr}>70 \%)$ segundo ensaios realizados em laboratório.

O solo B no primeiro estágio (Figura 3) permite observar uma diminuição de Q/Qo com o tempo e no segundo estágio (Figura 4) uma tendência linear, provavelmente, pelo pequeno diferencial de $\mathrm{H}$ entre um e outro estágio $(2 \mathrm{~cm})$. Assim, o regime permanente é facilmente alcançado devido ao pequeno volume de solo não saturado disponível para o segundo estágio. Também, se observou que, apesar de o solo ser de textura argila-siltosa, em ambos os estágios o tempo de equilíbrio foi consideravelmente rápido, em torno de 10 a $15 \mathrm{~min}$.

Por outro lado, similar aos resultados encontrados por Bell e Shofield (1990), em alguns casos obtiveram-se resultados anômalos, apresentando relações Q/Qo crescentes com o tempo que, no entanto, atingiram o regime permanente. Presumese que seja devido ao deslocamento gradual de ar ocluso pré-existente no solo pela introdução da água durante o ensaio.

Tabela 2: Ensaios de caracterização dos solos

Table 2: Tests for characterization of soils

\begin{tabular}{|c|c|c|}
\hline Local & A & B \\
\hline Profundidade $(\mathrm{m})$ & 0,45 & 0,45 \\
\hline Areia $(\%)$ & 91 & 12 \\
\hline Silte $(\%)$ & 4 & 29 \\
\hline Argila $(\%)$ & 5 & 59 \\
\hline $\mathrm{w}_{\mathrm{L}}(\%)$ & - & 53 \\
\hline $\mathrm{w}_{\mathrm{p}}(\%)$ & $\mathrm{NP}$ & 36 \\
\hline $\mathrm{I}_{\mathrm{p}}(\%)$ & $\mathrm{NP}$ & 17 \\
\hline$\rho_{\mathrm{s}}\left(\mathrm{g} / \mathrm{cm}^{3}\right)$ & 2,62 & 2,861 \\
\hline$\rho_{\mathrm{d}}\left(\mathrm{g} / \mathrm{cm}^{3}\right)$ & 1,77 & 1,203 \\
\hline $\mathrm{e}$ & 0,48 & 1,38 \\
\hline $\mathrm{SUCS}$ & $\mathrm{SC}$ & MH \\
\hline Classif. Granulométrica & Areia & Argila Siltosa \\
\hline
\end{tabular}


onde $\mathrm{w}_{\mathrm{L}}, \mathrm{w}_{\mathrm{p}}$ e representam os limites de liquidez e plasticidade, respectivamente; Ip, o índice de plasticidade; $\rho_{\mathrm{s}}$ e $\rho_{\mathrm{d}}$ as massas específicas dos sólidos e do solo seco respectivamente; e, índice de vazios; e SUCS representa as siglas do Sistema Unificado de Classificação dos Solos.

Após a determinação da condutividade hidráulica saturada de ambos os locais do estudo, foi efetuado um tratamento estatístico com a finalidade de caracterizar numericamente os resultados, visando identificar sua ocorrência quanto à distribuição da amostra, o que permite adotar o valor médio mais adequado em função dessa característica.

Para conhecimento da distribuição provável dos resultados de condutividade hidráulica saturada, foi empregado o teste de Kolmorogov-Smirnov (COSTA NETO, 1977). O teste foi realizado para os resultados de ensaios de condutividade hidráulica de ambos locais estudados sob duas hipóteses nulas. A primeira hipótese $\mathrm{H}_{0}$ afirma que a distribuição é normal e a segunda hipótese que segue uma distribuição log-normal.

Os testes estatísticos indicaram aceitação de ambas as hipóteses nulas para o nível de significância de 5\% (valor empregado em ensaios geotécnicos) em ambos os casos, ou seja, os resultados de condutividade hidráulica de ambos os locais seguem tanto uma distribuição normal quanto log-normal. Nas Figuras 5 e 6, são mostrados apenas os resultados obtidos para o teste da distribuição normal utilizando-se os resultados de $\mathrm{K}_{\mathrm{fs}}$ provenientes dos materiais A (um estágio) e B (segundo estágio), respectivamente.

Em função da distribuição normal dos resultados de condutividade hidráulica saturada, optou-se por empregar uma estatística paramétrica e caracterizar o valor médio dos resultados mediante média aritmética, ao invés de geométrica, sendo que esta última é a mais adequada quando se trata de uma distribuição log-normal (LEE et al., 1985). A Tabela 3 sintetiza os valores médios de $\mathrm{K}_{\mathrm{fs}}, \phi_{\mathrm{Gm}}$ e $\alpha$ dos resultados provenientes de ambos locais de estudo.

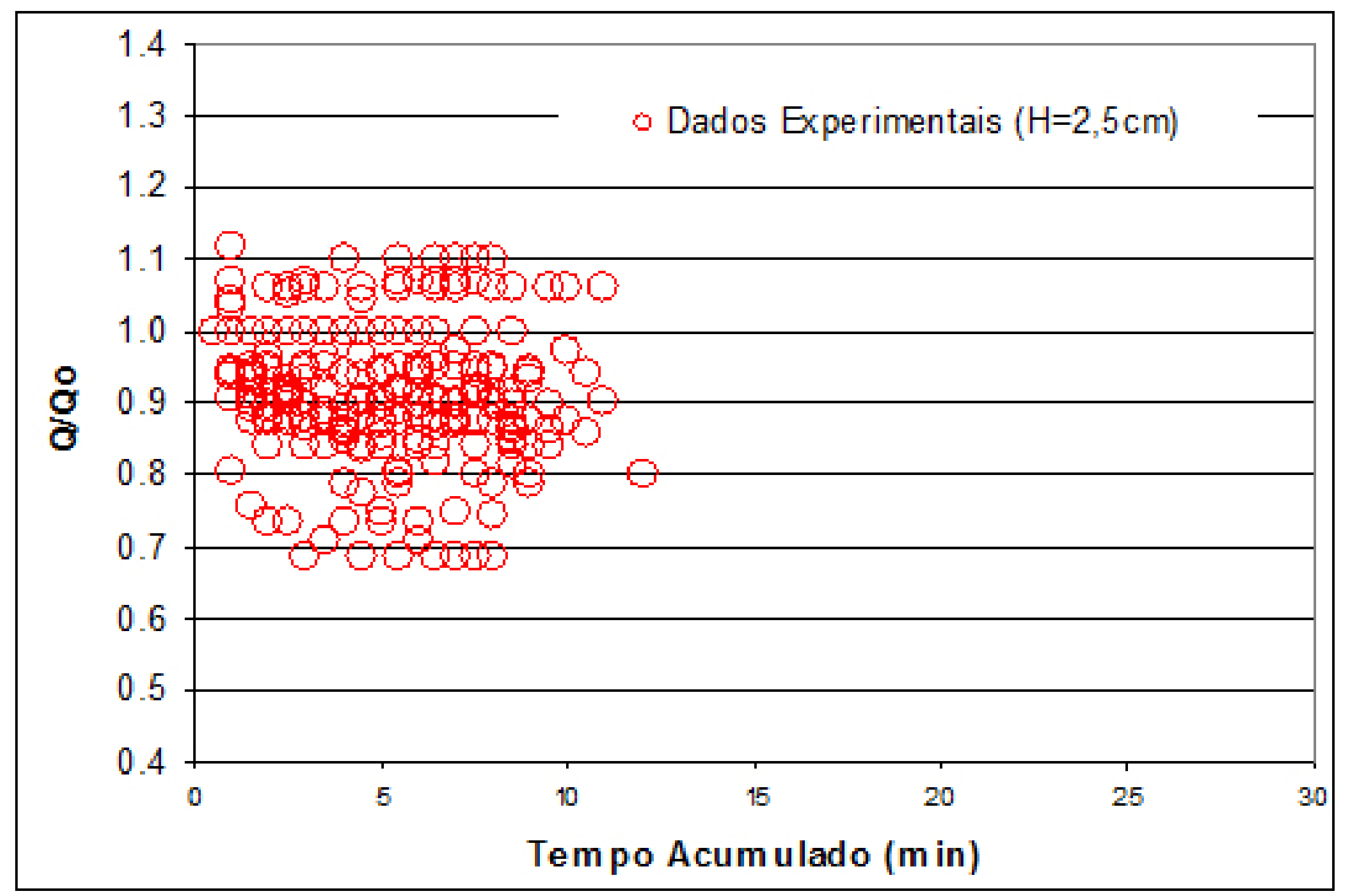

Figura 2: Relação $(Q / Q o)$ versus tempo resultante de ensaios no material $A(H=2,5 \mathrm{~cm})$.

Figure 2: Ratio $(Q / Q)$ versus time to the soil $A(H=2,5 \mathrm{~cm})$. 




Figura 3: Relação (Q/Qo) versus tempo resultante de ensaios no material B para o primeiro estágio $(H=10 \mathrm{~cm})$. Figure 3: Ratio $(Q / Q o)$ versus time to the soil $B$ with the first pond height $(H=10 \mathrm{~cm})$.

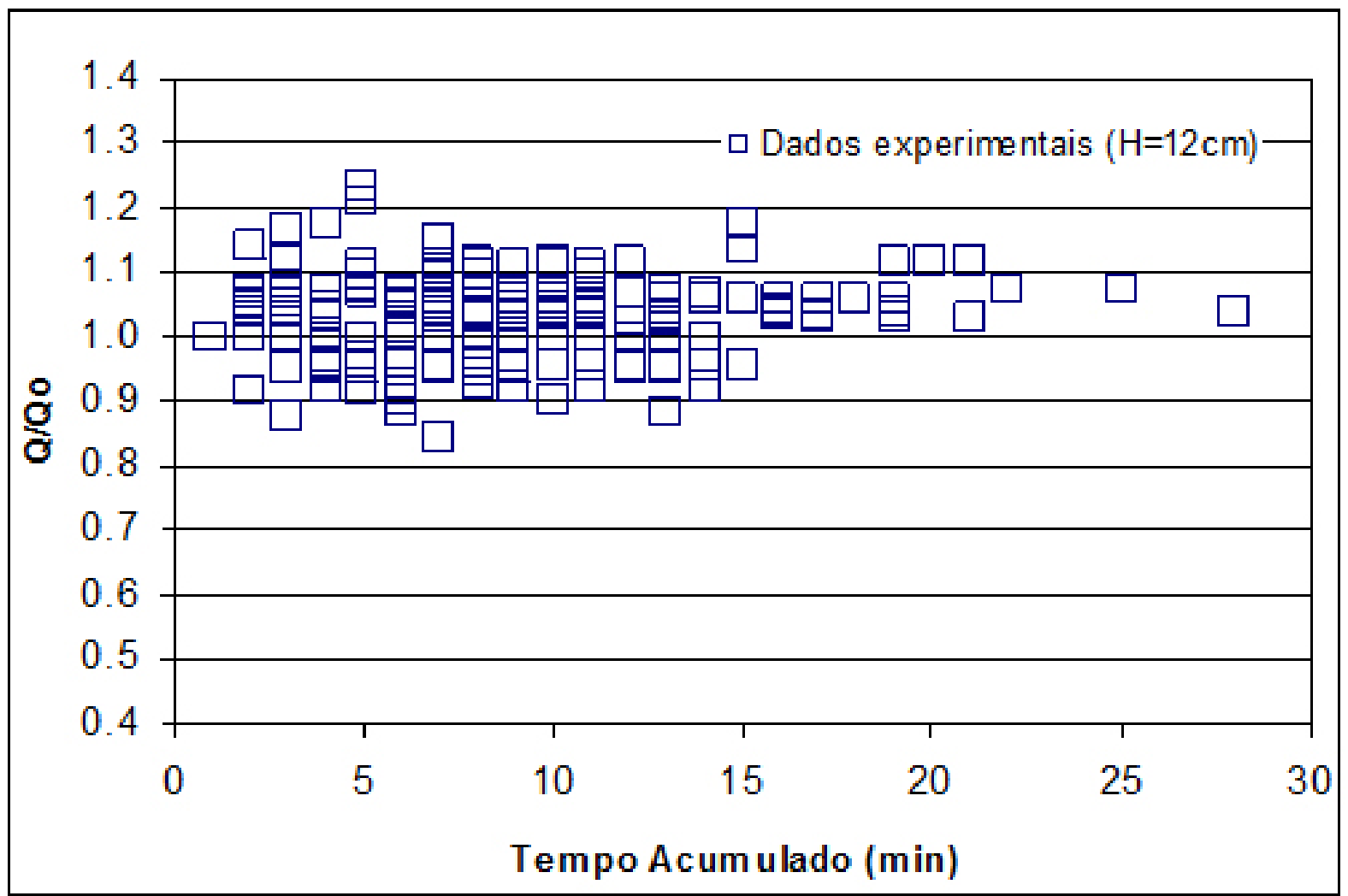

Figura 4: Relação $(Q / Q o)$ versus tempo resultante de ensaios no material B para o segundo estágio $(H=12 \mathrm{~cm})$.

Figure 4: Ratio $(Q / Q o)$ versus time to the soil $B$ with the second pond height $(H=12 \mathrm{~cm})$. 


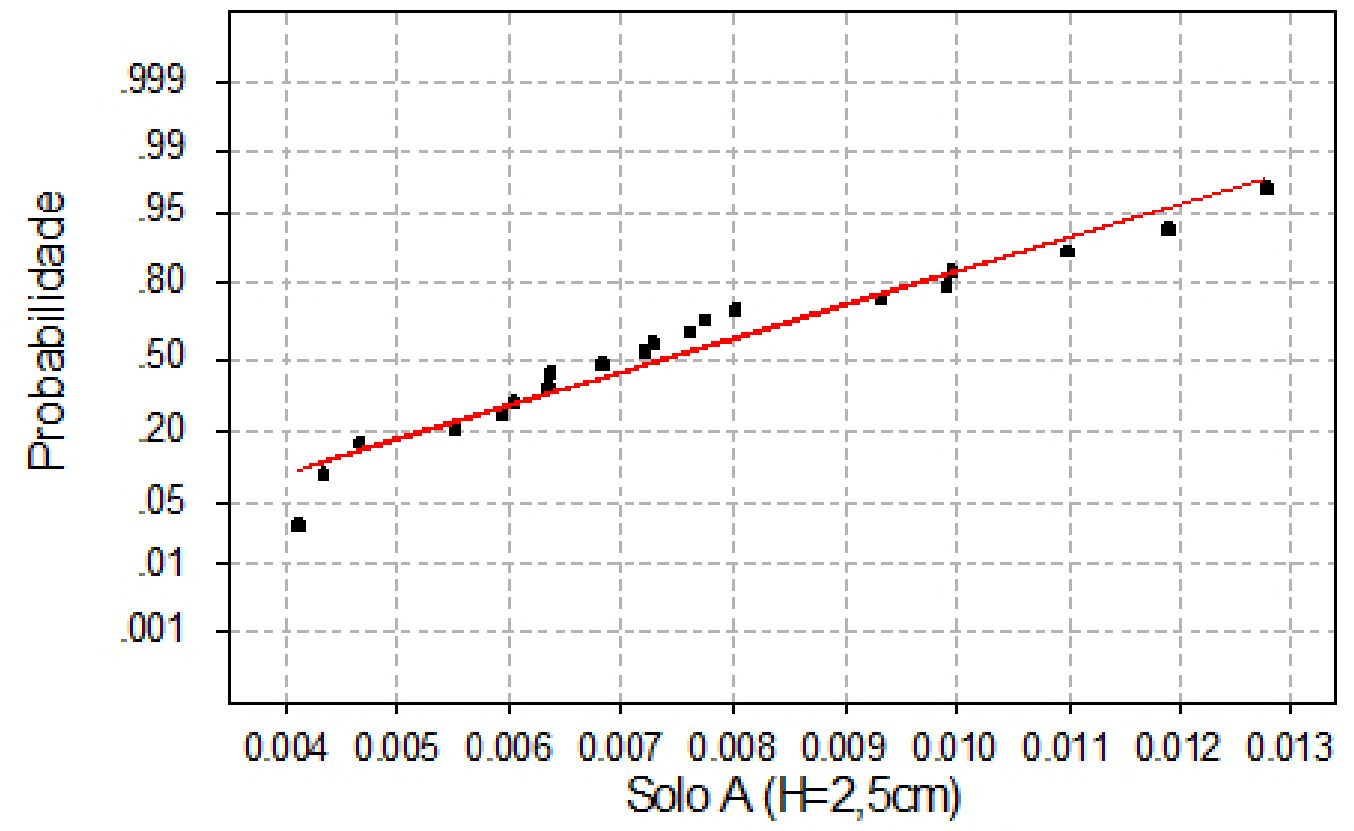

Aversge: 0.0074405

StDev. 0.0024884

KolmogororSmirnov Normality Test

N. 22

$D+: 0.133$ D: 0.088 D: 0.133

Appraximate $\mathrm{P}$-Value $>0.15$

Figura 5: Teste de Kolmorogov-Smirnov para verificação da distribuição normal nos resultados dos ensaios (um estágio) do material $A$.

Figure 5: Kolmorogov-Smirnov test to verify normal distribution of the results of tests (first pond height) of the soil A.



Aversge: 0.001731

KolmogorovSmirnov Normality Test D+: 0.093 D-: 0.081 D: 0.093

StDev. 0.0003885

Approximate $\mathrm{P}$-Value $>0.15$

Figura 6: Teste de Kolmorogov-Smirnov para verificação da distribuição normal nos resultados dos ensaios (segundo estágio) do material $B$.

Figure 6: Kolmorogov-Smirnov test to verify normal distribution of the results of tests (second pond height) of the soil A. 
Tabela 3: Valores médios de $\mathrm{K}_{\mathrm{fs}}, \phi_{\mathrm{Gm}}$ e $\alpha$ para cada tipo de solo e método de cálculo

Table 3: Calculation (average) of $\mathrm{K}_{\mathrm{fs}}$ and $\phi_{\mathrm{Gm}}$ with each approach and for each soil

\begin{tabular}{|c|c|c|c|c|c|c|}
\hline Parâmetro & \multicolumn{2}{|c|}{$\mathbf{K}_{\mathrm{fs}}$} & \multicolumn{2}{c|}{$\varphi_{\text {Gms }}$} & \multicolumn{2}{c|}{$\alpha$} \\
\hline Local & A & B & A & B & A & B \\
\hline Profundidade & 0,40 & 0,40 & 0,40 & 0,40 & 0,40 & 0,40 \\
\hline Resultados negativos & - & 2 & - & 3 & - & 5 \\
\hline Um estágio & $8,6 \times 10^{-3}$ & $1,7 \times 10^{-3}$ & $8,4 \times 10^{-2}$ & $1,1 \times 10^{-2}$ & $0,20^{(1)}$ & $0,16^{(2)}$ \\
\hline C.V & 33,2 & 21,3 & 34,1 & 21,3 & 0 & $0-$ \\
\hline Dois estágios & - & $1,6 \times 10^{-3}$ & - & $1,4 \times 10^{-2}$ & - & $0,16^{(3)}$ \\
\hline C.V & - & 92,0 & - & 46,1 & - & 126,9 \\
\hline
\end{tabular}

Dos resultados da Tabela 3, observa-se que, devido a sua textura, o solo argilo-siltoso B deveria possuir uma condutividade hidráulica relativamente menor em relação ao material arenoso A. No entanto, seu elevado índice de vazios (Tabela 2 ) e macroporosidade (inferida da Tabela 1 e igual a $\alpha=0,16 \mathrm{~cm}^{-1}$ ) mostram que se trata de uma argila porosa.

Adicionalmente, no solo B obteve-se na sua totalidade 2 resultados negativos de $\mathrm{K}_{\mathrm{fs}}$ e 3 de $\phi_{\mathrm{Gm}}$. Esses resultados são comuns quando se utiliza o método dos dois estágios e devido aos fatores já comentados anteriormente. Por essa razão, optouse por realizar análises destinadas a elucidar esses fatos.

Análises da variação dos resultados pela obtenção de $K_{\mathrm{fs}}$ pelo emprego do método de dois estágios num mesmo furo de sondagem

Como já mencionado anteriormente, a técnica dos dois estágios envolve um condicionamento inapropriado para obtenção do parâmetro $\mathrm{K}_{\mathrm{fs}}, \phi_{\mathrm{Gm}}$ (e, consequentemente, de $\alpha$ ), quando utilizada a solução das equações simultâneas a partir da expressão (1).

Em virtude do interesse em estabelecer a funcionalidade da utilização dessa técnica, principalmente para determinação de $\mathrm{K}_{\mathrm{fs}}$ foram realizadas análises envolvendo a determinação do erro da medição, da faixa de valores positivos e intervalo de confiança decorrentes desta técnica.

A primeira análise diz respeito à determinação do erro da medição empregado por Philips (1985), aplicada para a expressão (1) de Reynolds e Elrick (1985). Assim, é obtido um erro fracional da condutividade $\mathrm{K}_{\mathrm{fs} 2}$ proveniente de dois estágios em relação à condutividade $\mathrm{K}_{\mathrm{fs} 1}$ para um estágio.

Para isso ser possível, na análise foram empregados dados de ensaio do solo $\mathrm{B}$, tais como: $\mathrm{H}_{1}=10 \mathrm{~cm}, \mathrm{H}_{2}=12 \mathrm{~cm}, \mathrm{a}_{1}=\mathrm{a}_{2}=3 \mathrm{~cm}, \mathrm{C}_{1}=1,30$, $\mathrm{C}_{2}=1,47$, com o conhecimento de $\alpha_{1}=0,16 \mathrm{~cm}^{-}$ ${ }^{1}$ (igual ao valor médio de $\alpha_{2}$ ) e considerando homogeneidade do solo sendo $\mathrm{K}_{\mathrm{fs} 1}=\mathrm{K}_{\mathrm{fs} 2}$.

Denominando-se $\delta$ e $\varepsilon$ aos erros provenientes das medições de $Q_{1}$ e $Q_{2}$, têm-se, a partir da equação (1), as expressões seguintes:

$$
\begin{aligned}
& 813,66 \mathrm{~K}_{\mathrm{fs} 1}(1+\delta)=511,60 \mathrm{~K}_{\mathrm{fs} 2}+48,33 \phi_{\mathrm{Gm} 2} \\
& 997,32 \mathrm{~K}_{\mathrm{fs} 1}(1+\varepsilon)=665,44 \mathrm{~K}_{\mathrm{fs} 2}+53,10 \phi_{\mathrm{Gm} 2}
\end{aligned}
$$

A partir de (5) têm-se $\phi_{\mathrm{Gm}}=\mathrm{K}_{\mathrm{fs} 2} / \alpha$ e, adotando $\gamma=(1+\delta) /(1+\varepsilon)$, obtemos:

$$
\alpha=0,0798 \frac{(1,1154-\gamma)}{(\gamma-0,9422)}
$$

$$
\frac{\mathrm{K}_{\mathrm{fs} 2}}{\mathrm{~K}_{\mathrm{fs} 1}}=8,6517(1+\varepsilon)(1,1154-\gamma)
$$

Das equações (11) e (12) resulta que:

$$
\begin{gathered}
\alpha \mathrm{e}_{\mathrm{fs} 2}=0 \quad \text { se } \gamma=1,1154 \\
\alpha<0 \text { e }_{\mathrm{fs} 2}<\quad \text { se } \gamma>1,1154 \\
\alpha<0 \quad \text { se } \gamma<0,9422
\end{gathered}
$$

\footnotetext{
(1) valor inferido a partir da avaliação visual do material;

(2) valor propositalmente igual ao a médio medido pelos dois estágios;

(3) valor medido;

C.V coeficiente de variação.
} 
$\alpha=\infty \quad$ se $\gamma=0,9422$

Logo para $\gamma=1+\kappa$, com $\varepsilon$ e $\kappa$ próximos a zero, o erro da relação $\mathrm{K}_{\mathrm{fs} 2} / \mathrm{K}_{\mathrm{fs} 1}$ será $-8,6517 \kappa \mathrm{ou}$, ainda, para o intervalo $0,9885 \leq \gamma \leq 1,0115$, sendo que:

$$
0,90 \leq \mathrm{K}_{\mathrm{fs} 2} / \mathrm{K}_{\mathrm{fs} 1} \leq 1,10
$$

Da relação (17) conclui-se que $\mathrm{K}_{\mathrm{fs} 2}$ será exato para uma variação de aproximadamente $\pm 10,0 \%$, faixa que permite satisfazer a equação (1) para os dados do solo B.

Apenas um resultado foi obtido dentro desta faixa $\left(\mathrm{K}_{\mathrm{fs} 2} / \mathrm{K}_{\mathrm{fs} 1}=0,93\right)$ dentre os 20 ensaios realizados. Isto mostra que, na prática, a hipótese de isotropia, isto é $\mathrm{K}_{\mathrm{fs} 2} / \mathrm{K}_{\mathrm{fs} 1}=1$ e $\phi_{\mathrm{GM} 2} / \phi_{\mathrm{GM} 1}=1$ é dificilmente satisfeita por tratar-se de solos indeformados, cuja heterogeneidade é natural no meio poroso. A hipótese da isotropia é um requisito para solução das equações simultâneas no método das duas alturas.

Apesar de alguns resultados de $\mathrm{K}_{\mathrm{fs}}$ não se adequarem a essa condição, não podemos descartar a possibilidade dessa técnica, pois existem outros resultados que podem ser representativos do local A de estudo. Por essa razão, se realizou uma segunda análise, onde se determinou estatisticamente o intervalo de confiança para um nível de significância de 5\% de cada valor médio amostral de $\mathrm{K}_{\mathrm{fs} 1}$ e $\mathrm{K}_{\mathrm{fs} 2 \text {, }}$ resultando:

$$
\begin{aligned}
& \mathrm{K}_{\mathrm{fs} 1}=1,73 \times 10^{-3} \pm 1,73 \times 10^{-4} \mathrm{~cm} / \mathrm{s} \\
& \mathrm{K}_{\mathrm{fs} 2}=1,61 \times 10^{-3} \pm 7,60 \times 10^{-4} \mathrm{~cm} / \mathrm{s}
\end{aligned}
$$

Arranjando as expressões (18) e (19) para obter os valores mínimos e máximos para a relação $\mathrm{K}_{\mathrm{fs} 2} /$ $\mathrm{K}_{\mathrm{fs} 1}$ resulta:

$$
0,445<\mathrm{K}_{\mathrm{fs} 2} / \mathrm{K}_{\mathrm{fs} 1}<1,519
$$

Com isto, pode-se estabelecer a faixa de variação em que esses parâmetros se encontram, sendo que 7 dos 20 ensaios realizados mostraramse nesse intervalo e, como esperado, também $\mathrm{o}$ único valor $\left(\mathrm{K}_{\mathrm{fs} 2} / \mathrm{K}_{\mathrm{fs} 1}=0,93\right)$ que se adequou à equação (1) na análise anterior.

Apesar das limitações da obtenção dos parâmetros $\mathrm{K}_{\mathrm{fs}}$ e $\phi_{\mathrm{GM}}$ pela técnica de dois estágios, quase a metade dos ensaios encaixaram-se nesse intervalo. Isto é favorável, pois é preferível a obtenção desses parâmetros pela medição direta, que é possível desde que sejam levadas em consideração as limitações para sua obtenção.
Valores de $\mathrm{K}_{\mathrm{fs} 2} / \mathrm{K}_{\mathrm{fs} 1}$ fora dessa faixa de variação não são representativos (para o nível de significância adotado igual a 5\%) ou também podem ser incoerentes, mostrando valores negativos devido às limitações da técnica, que ocorrem comumente na prática (valores negativos de $\mathrm{K}_{\mathrm{fs}}$ e $\left.\phi_{\mathrm{Gm}}\right)$. Sendo assim, a terceira análise consistiu em investigar os limites em que $\mathrm{K}_{\mathrm{fs} 2} / \mathrm{K}_{\mathrm{fs} 1}$ são positivos contendo valores representativos $\mathrm{e}$ não representativos da população.

Segundo Reynolds e Elrick (1986), a determinação de $\mathrm{K}_{\mathrm{fs}}$ e $\phi_{\mathrm{Gm}}$ proveniente de ensaios com dois estágios pode ser obtida da relação (8):

$$
\mathrm{K}_{\mathrm{fs}}=\mathrm{G}_{2} \mathrm{Q}_{2}-\mathrm{G}_{1} \mathrm{Q}_{1}
$$

$$
\phi_{\mathrm{Gm}}=\mathrm{J}_{1} \mathrm{Q}_{1}-\mathrm{J}_{2} \mathrm{Q}_{2}
$$

Onde:

$$
\begin{gathered}
\mathrm{G}_{1}=\frac{\mathrm{G}_{2} \mathrm{H}_{2} \mathrm{C}_{1}}{\mathrm{H}_{1} \mathrm{C}_{2}} \\
\mathrm{G}_{2}=\frac{\mathrm{H}_{1} \mathrm{C}_{1}}{\pi\left(2 \mathrm{H}_{1}+\mathrm{H}_{2}\left(\mathrm{H}_{2}-\mathrm{H}_{1}\right)+\mathrm{a}^{2}\left(\mathrm{H}_{1} \mathrm{C}_{2}-\mathrm{H}_{2} \mathrm{C}_{1}\right)\right)} \\
\mathrm{J}_{1}=\frac{\left(2 \mathrm{H}_{2}{ }^{2}+\mathrm{a}^{2} \mathrm{C}_{2}\right) \mathrm{C}_{1}}{2 \pi\left(2 \mathrm{H}_{1} \mathrm{H}_{2}\left(\mathrm{H}_{2}-\mathrm{H}_{1}\right)+\mathrm{a}^{2}\left(\mathrm{H}_{1} \mathrm{C}_{2}-\mathrm{H}_{2} \mathrm{C}_{1}\right)\right)} \\
\mathrm{J}_{2}=\mathrm{J}_{1} \frac{\left(2 \mathrm{H}_{1}{ }^{2}+\mathrm{a}^{2} \mathrm{C}_{1}\right) \mathrm{C}_{2}}{\left(2 \mathrm{H}_{2}{ }^{2}+\mathrm{a}^{2} \mathrm{C}_{2}\right) \mathrm{C}_{1}}
\end{gathered}
$$

Para as mesmas características do ensaio (i.e., valores de $\mathrm{H}$, a, $\mathrm{C}$ e $\alpha$ ) empregadas na análise anterior, substituídos em (21) e (22) obtemos:

$$
\begin{aligned}
& \mathrm{K}_{\mathrm{fs}}=0,009670 \mathrm{Q}_{2}-0,010624 \mathrm{Q}_{1} \\
& \phi_{\mathrm{Gm}}=0,133140 \mathrm{Q}_{1}-0,102360 \mathrm{Q}_{2}
\end{aligned}
$$

Dessas expressões, resulta que:

$\mathrm{K}_{\mathrm{fs}}<0 \quad$ se $\mathrm{Q}_{2} / \mathrm{Q}_{1}>\mathrm{G} 1 / \mathrm{G} 2$ ou $\mathrm{Q}_{2} / \mathrm{Q}_{1}>1,099$ 
$\phi_{\mathrm{Gm}}<0 \quad$ se $\mathrm{Q}_{2} / \mathrm{Q} 1<\mathrm{J} 1 / \mathrm{J} 2$ ou $\mathrm{Q} 2 / \mathrm{Q} 1<1,301$

Das expressões (29) e (30) deduzimos que $\mathrm{K}_{\mathrm{fs}}$ e $\phi_{\mathrm{Gm}}$ serão positivos se:

$$
1,099<\mathrm{Q}_{2} / \mathrm{Q}_{1}<1,301
$$

Ainda, a relação (31) pode ser expressa em termos de condutividade hidráulica, onde $\mathrm{Q}_{2}$ e $\mathrm{Q}_{1}$ foram empregados na determinação de $\mathrm{K}_{\mathrm{fs} 2}$ e $\mathrm{Q}_{1}$, na determinação de $\mathrm{K}_{\mathrm{fs} 1}$, o que resulta:

$$
0<\mathrm{K}_{\mathrm{fs} 2} / \mathrm{K}_{\mathrm{fs} 1}<1,589
$$

Da expressão (32), a relação $\mathrm{K}_{\mathrm{fs} 2} / \mathrm{K}_{\mathrm{fs} 1}$ poderá fornecer resultados coerentes até um valor máximo de 1,59 (valores maiores produzem resultados negativos de $\phi_{\mathrm{Gm} 2}$ ), sendo que neste trabalho, 15 de 20 ensaios mostraram resultados dentro desta faixa de variação, sendo cinco deles negativos.

Os cinco resultados representam os $25 \%$. Esta porcentagem é inferior se comparada aos resultados obtidos por outros autores, entre eles Reynolds e Elrick (1985). Segundo esses autores, os valores negativos podem oscilar entre 47 a $68 \%$ em solos com textura (silte a argila) e macroporosidade (muito elevada numa argila porosa) com características similares aos do solo B. Presume-se que essa menor quantidade de resultados negativos seja produto de intensivos cuidados no procedimento experimental, desde a execução do furo de sondagem evitando a compactação do solo, impedindo o fechamento dos poros das paredes do furo por atrito com o trado, preservando a forma cilíndrica do furo, não realizando ensaios em camadas muito superficiais onde a heterogeneidade causada pelas raízes das plantas ou insetos possa ser maior, aplicação de vácuo no reservatório para evitar quedas intempestivas de água no furo de sondagem no início do ensaio, etc.

Análises da variação dos resultados pela obtenção de $K_{\mathrm{fs}}$ pelo emprego do método de um estágio

Segundo Elrick et al. (1989), a adoção do parâmetro $\alpha$ para o método de um estágio é possível, uma vezque não influisignificativamente na determinação de $\mathrm{K}_{\mathrm{fs}}$ e $\phi_{\mathrm{GM}}$.

Com base nessas hipóteses, optou-se por verificar como variam os resultados da condutividade hidráulica, quando adotado o valor $\alpha=0,16 \mathrm{~cm}^{-1}$ no método de um estágio $\left(\mathrm{K}_{\mathrm{fs}}\right.$ dos materiais A e B mostrados na Tabela 3) e quando empregados outros valores de $\alpha$ numa

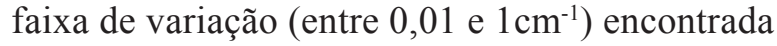
por diversos autores em diversos tipos de solos e ensaios. A Figura 7 ilustra os resultados de $\mathrm{K}_{\mathrm{fs}}$ versus $\alpha$, para o material $\mathrm{B}$ e, adicionalmente, o intervalo de confiança do valor médio de $\mathrm{K}_{\mathrm{fs}}$ quando $\alpha=0,16 \mathrm{~cm}^{-1}$ mostrado na expressão (18).

Da Figura 7 observa-se que, a dispersão dos resultados de $\mathrm{K}_{\mathrm{fs}}$ aumenta com o crescimento do parâmetro $\alpha$. Contudo, muitos valores de $\mathrm{K}_{\mathrm{fs}}$ situam-se entre o intervalo de confiança e quando $\alpha$ varia entre $0,10 \mathrm{e} 1 \mathrm{~cm}^{-1}$. Este comportamento mostrou-se similar para os resultados do material A.

A dispersão dos resultados de $\mathrm{K}_{\mathrm{fs}}$ foi quantificada com a determinação do desvio padrão para diferentes valores de $\alpha$. Esses resultados são mostrados na Figura 8 para os materiais A e B, onde se observa que a dispersão dos resultados é maior não somente devido ao aumento do parâmetro $\alpha$, mas também, pelo tipo de material. Assim, o material A, de textura arenosa, forneceu maior dispersão em relação aos resultados de $\mathrm{K}_{\mathrm{fs}}$ do material argilo-siltoso B. 


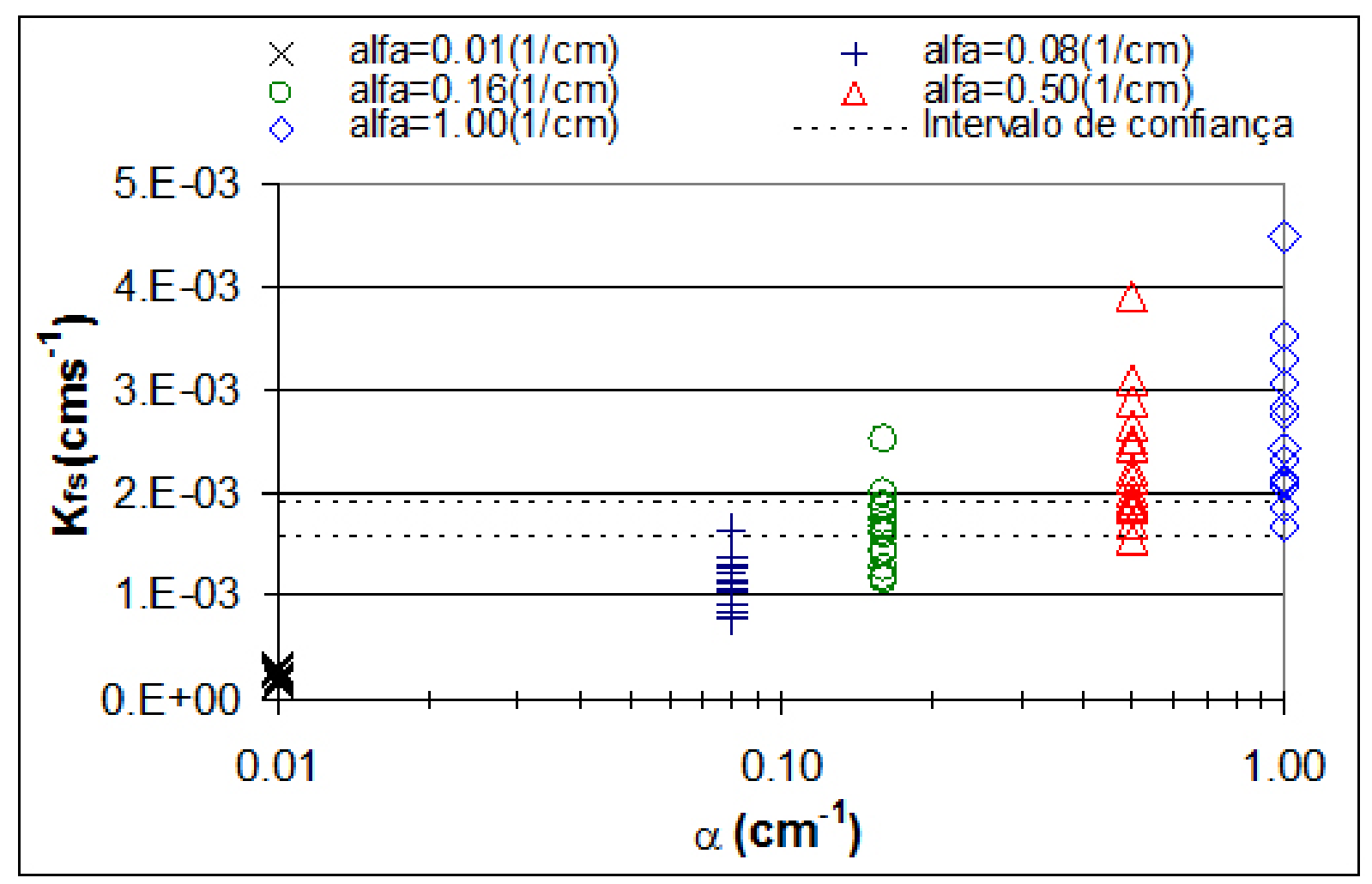

Figura 7: Valores de $K_{f s}$ versus a para o material B obtido pelo método de um estágio.

Figure 7: $K_{f s}$ versus $\alpha$ for the soil $B$ calculated with the one pond height approach.



Figura 8: Desvio padrão de $K_{\text {fs }}$ versus a para os materiais A e B obtidos pelo método de um estágio.

Figure 8: Standard deviation $K_{f s}$ versus $\alpha$ for soils $A$ and $B$ calculated with the one pond height approach. 


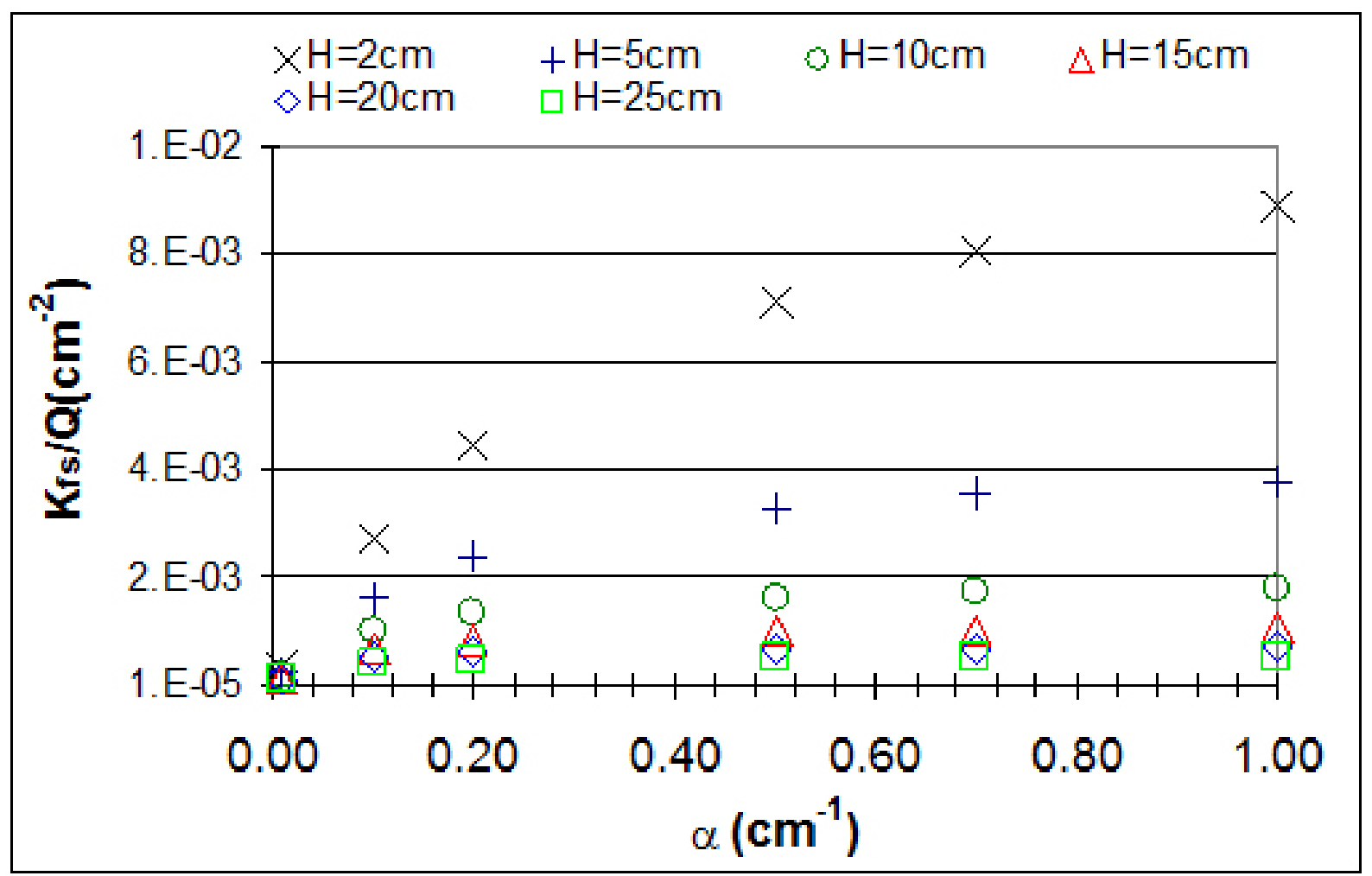

Figura 9: $K_{f s} / Q$ versus $\alpha$ para diferentes alturas $H$.

Figure 9: $K_{f s} / Q$ versus $\alpha$ for different pond heights.

No entanto, essa variabilidade não pode ser totalmente atribuída à textura do material, pois a altura constante $\mathrm{H}$ empregada para ambos os materiais durante os ensaios poderia também influenciar nesses resultados. Para os ensaios foram empregadas as alturas de $2,5 \mathrm{~cm}$ e $10 \mathrm{~cm}$ nos materiais $\mathrm{A}$ e $\mathrm{B}$, respectivamente.

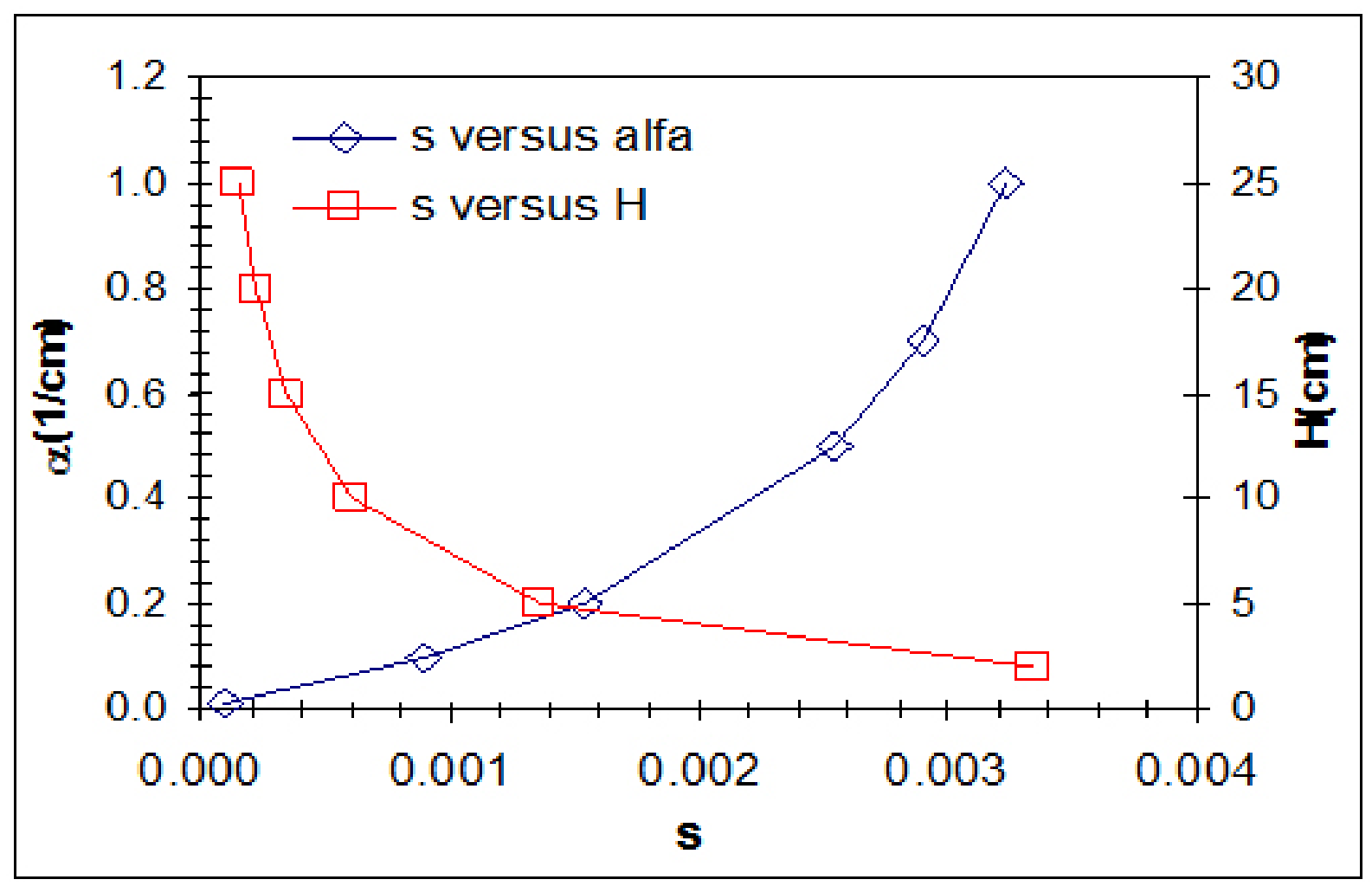

Figura 10: Desvio padrão $(s)$ de $K_{f s} / Q$ em relação à altura $H$ e $\alpha$.

Figure 10: Standard deviation (s) of $K_{f_{s}} / Q$ as function of height $H$ and $\alpha$. 
Com o intuito de verificar essa variabilidade para qualquer tipo de solo, a equação (6) foi modificada resultando na expressão:

$$
\mathrm{K}_{\mathrm{fs}} / \mathrm{Q}=\frac{\mathrm{C}}{\left(2 \pi \mathrm{H}^{2}+\pi \mathrm{a}^{2} \mathrm{C}+2 \pi \mathrm{H} / \alpha\right)}
$$

Onde: $\mathrm{a}=3 \mathrm{~cm} ; \mathrm{H}=2,5 ; 10 ; 15 ; 20$ e $25 \mathrm{~cm}$; $\alpha=0,01 ; 0,1 ; 0,2 ; 0,5 ; 0,7$ e $1,0 \mathrm{~cm}^{-1}$.

Assim, a partir dessa equação de fluxo, é possível observar a influência da variação do parâmetro $\alpha$ e adicionalmente da altura $\mathrm{H}$. A Figura 10 mostra esses resultados.

Tal como observado nessa figura, ocorre uma dispersão crescente de $\mathrm{K}_{\mathrm{fs}} / \mathrm{Q}$ com o aumento do $\alpha$ e para alturas $\mathrm{H}$ cada vez menores. No gráfico resulta evidente que valores abaixo de $\alpha=20 \mathrm{~cm}^{-1} \mathrm{e}$ superiores a $\mathrm{H}=5 \mathrm{~cm}$ aproximadamente, fornecerão dispersões mais moderadas dentro da faixa avaliada.

\section{Considerações para uso das técnicas de um e dois estágios}

O método do permeâmetro Guelph destaca-se pela facilidade de uso, praticidade para obtenção dos dados experimentais e, principalmente, por possuir a mais aceita fundamentação teórica para inclusão dos efeitos matriciais na determinação de $\mathrm{K}_{\mathrm{fs}}, \phi_{\mathrm{Gm}} \mathrm{e}$, consequentemente, de $\alpha$. É inegável que a técnica de dois estágios (REYNOLDS e ELRICK., 1985) possui limitações na obtenção desses parâmetros. Apesar disso, é o único método que permite a medição direta deles e poderá ser empregado desde que se leve em consideração as limitações aqui observadas.

Em casos em que seja viável, poderá ser utilizado o método de dois estágios empregando um estudo estatístico. $\mathrm{O}$ procedimento consiste em caracterizar hidraulicamente um meio poroso com um número de ensaios maior ou igual ao necessário para obter uma representatividade estatística (se sugere não menos de 30). De posse dos resultados, deverá ser avaliado o tipo de distribuição, pois, disso dependerá o tipo de estatística a ser empregada (paramétrica ou não). Geralmente, a condutividade hidráulica segue uma distribuição log-normal e/ou normal. Uma vez identificada sua distribuição, serão considerados como representativos os resultados de ensaios, que tratados estatisticamente se encontrem dentro do intervalo de confiança para um nível de significância de 5\%.

Adicionalmente, caso se deseje a determinação de um número exato de ensaios para a caracterização hidráulica, este poderá ser calculado a partir de um experimento piloto que consistirá na execução de um número reduzido de ensaios (maior que 10) dos quais será obtido o coeficiente de variação. Com o coeficiente de variação (C.V) do experimento piloto e adotando um erro máximo $(\Delta \mathrm{r})$ esperado de $\mathrm{K}_{\mathrm{fs}}$, poderemos obter o valor $\mathrm{t}_{\alpha}$ da função $t$ student, a partir da equação abaixo.

$$
\mathrm{t}_{\alpha}=\frac{\Delta \mathrm{r}}{\mathrm{C} . \mathrm{V}}
$$

Tabelas estatísticas permitem a obtenção do número $n$ mínimo de amostras em função do valor $\mathrm{t}_{\alpha}$ student para um determinado nível de significância $\alpha(10 \%, 5 \%, 1 \%)$, sendo preferível adotar $\alpha=5 \%$ por ser utilizado satisfatoriamente em testes estatísticos com fins geotécnicos (RÉTTHÁTI, 1988).

Quanto à técnica de um estágio (ELRICK et al., 1989), poderá ser empregada em um número reduzido de determinações com satisfatória aproximação da condutividade hidráulica saturada, porém, quando não seja de interesse o conhecimento de $\alpha$ e $\phi_{\mathrm{Gm}}$ do solo.

Para minimizar erros indesejáveis na determinação de $\mathrm{K}_{\mathrm{fs}}$ nesta técnica, será necessário levar algumas considerações na escolha da altura de carga constante $\mathrm{H}$ no furo de sondagem e de $\alpha$ a serem empregadas. Assim, serão preferíveis (desde que a capacidade do reservatório do permeâmetro o permita) maiores alturas de água $\mathrm{H}$. Quanto ao parâmetro $\alpha$, caso não exista subsídios para sua medição por algum outro método experimental, poderá ser adotado um valor inicial entre $0,1 \mathrm{e} 0,2 \mathrm{~cm}^{-1}$.

\section{CONCLUSÕES}

Este artigo abordou aspectos que devem ser levados em conta na medição da condutividade hidráulica saturada determinada in situ com uso do método do permeâmetro Guelph.

A partir desses resultados, conclui-se que, dada a importância do método de dois estágios na determinação direta de $\mathrm{K}_{\mathrm{fs}}$ e $\phi_{\mathrm{Gm}}$ (conseqüentemente, de $\alpha$ ), será possível sua utilização desde que se leve em conta suas limitações teóricas e experimentais. Ainda, os resultados poderão ser mais representativos sendo empregado um número adequado de ensaios previamente estipulados (se sugere acima de 30 ), ou calculados, com a realização prévia de um 
experimento piloto que permita obter resultados representativos estatisticamente (como o método sugerido anteriormete). Em contrapartida, o método de um estágio poderá ser aplicado visando boa determinação da condutividade hidráulica e com número reduzido de ensaios, levando em conta que, para esse objetivo, será preferível gerar bulbos de umidade maiores no solo (maior $\mathrm{H}$ ) e adotar valores de $\alpha$ entre 0,1 e $0,2 \mathrm{~cm}^{-1}$.

\section{AGRADECIMENTOS}

Os autores agradecem à FUNDUNESP, CNPq e PETROBRAS pelo apoio financeiro para elaboração desta pesquisa.

\section{REFERÊNCIAS BIBLIOGRÁFICAS}

AMOOZEGAR, A. Comparison of the Glover solution with the simultaneous equations approach for measuring hydraulic conductivity. Soil Science Society of America Journal, v. 53, n 5, 1989. p. 13621367.

BAGARELLO, V.; PROVENZANO, G. Factors affecting field and laboratory measurement of saturated hydraulic conductivity. Transactions of the ASAE, v. 39, nº1, 1996. p. 153-159.

BANTON, O. Field and laboratory-determined hydraulic conductivities considering anisotropy ad core surface area. Soil Sci. Soc. of Am. v.57 (1), 1993. p. $10-15$.

BELL, R. W.; SHOFIELD, N. J. Design and application of a constant head well permeameter for shallow high saturated hydraulic conductivity. Hydrol. Processes, v. 4, 1990. p. 327-342.

BRESLER, E. Analysis of trickle irrigation with application to design problems. Irrig.Sci., v.1, 1978. p.3-17.

COSTANETO, P. L. O. Estatística. Editora Edgard Blücher. São Paulo-SP, 1977.

ELRICK, D. E.; REYNOLDS, W. D.; TAN, K. A. Hydraulic conductivity measurements in the unsaturated zone using improved well analysis. Groundwater Monitoring Review. v.9, 1989. p.184193.

GARDNER, W. R. Some steady-state solutions of the unsaturated moisture flow equation with application to evaporation from a water table. Soil Science, Baltimore, v.85, 1958. p.228-232.

LEE, D. M.; REYNOLDS, W. D.; ELRICK, D. E.; CLOTHIER B.E. A comparison of three techniques for measuring saturated hydraulic conductivity. Canadian Journal of Soil Science. v.65, no .3, 1985. p.563-573.

PHILIP, J. R. Approximate analysis of the borehole permeameter in unsaturated soil - Water. Resources Research. v. 21, n7, 1985. p.1025-1033.

RAGAB, R.; COOPER J. D. Variability of unsaturated zone water transport parameters: Implications for hydrological modelling.1. In situ measurements. Journal of Hydrology. v.148, 1990. p.109-131.

RÉTTHATI, L. Probabilistics solutions in geotechnics. Elsevier, New York, 1988. 451p.

REYNOLDS, W. D.; ELRICK, D. E. A method for simultaneous in situ measurement in the vadose zone of field saturated hydraulic conductivity, sorptivity and the conductivity-pressure head relationships. Ground Water Monitoring. Rev. v.6(1), 1986. p.84-95.

REYNOLDS, W. D.; ELRICK, D. E. In situ measurement of field saturated hydraulic conductivity, sorptivity and the $\alpha$-parameter using the Guelph permeameter. Soil Science, v.140, n ${ }^{4}$, 1985. p. 292302.

REYNOLDS, W. D.; ELRICK, D. E.; TOPP, G. C. A reexamination of the constant head well permeameter method for measuring saturated hydraulic conductivity above the water table. Soil Science, v.136, 1983. p.250-268.

RICHARDS, B. G. Capillary conduction of liquids through porous medium. Physics, New York, 1, 1931. p. 318-333.

SCOOTER, D. R.; CLOTHIER, B. E.; HARPER, E. R. Measuring saturated hydraulic conductivity and sorptivity using twin rings. Aust. Journal Soil Res. v.20, 1982. p. 295-304.

SOILMOISTURE EQUIPMENT CORP. Guelph Permeameter. Operating - Instructions, Soilmoisture manual, Rev.8, 1986. p.1-25.

SOTO, M. A. A. Estudo da condutividade hidráulica em solos não saturados, Dissertação de Mestrado, Universidade de São Paulo (USP), Brasil, 1999. 123p.

WHITE, I.; SULLY, M. J. Macroscopic and microscopic capillary length and time scales from field infiltration. Water Resources Research, v. $23, \mathrm{n}^{\circ} 8$, 1987. p. 1514-1522. 
